\title{
Transapical transcatheter mitral valve implantation in mitral annular calcification: Role of fusion imaging
}

Amedeo Anselmi, MD, PhD, ${ }^{\mathrm{a}}$ Hervé Corbineau, MD, ${ }^{\mathrm{a}}$ Jean-Philippe Verhoye, $\mathrm{MD}, \mathrm{PhD},{ }^{\mathrm{a}}$ and Erwan Donal, MD, PhD, ${ }^{\mathrm{b}}$ Rennes, France

\footnotetext{
From the ${ }^{\mathrm{a} D i v i s i o n}$ of Thoracic and Cardiovascular Surgery, Pontchaillou University Hospital; and ${ }^{\mathrm{b}}$ Division of Cardiology, Pontchaillou University Hospital, Rennes, France.

Disclosures: Dr Anselmi discloses an association with Abbott Inc., including proctoring, consultancy, and lecture fees. All other authors reported no conflicts of interest.

The Journal policy requires editors and reviewers to disclose conflicts of interest and to decline handling or reviewing manuscripts for which they may have a conflict of interest. The editors and reviewers of this article have no conflicts of interest.

Received for publication June 8, 2020; revisions received June 8, 2020; accepted for publication June 17, 2020; available ahead of print June 25, 2020.

Address for reprints: Amedeo Anselmi, MD, PhD, Division of Thoracic and Cardiovascular Surgery, Pontchaillou University Hospital, Rennes 35000, France (E-mail: amedeo.anselmi@chu-rennes.fr).

JTCVS Techniques 2020;3:146-9

2666-2507

Copyright (C 2020 The Authors. Published by Elsevier Inc. on behalf of The American Association for Thoracic Surgery. This is an open access article under the CC BY-NC-ND license (http://creativecommons.org/licenses/bync-nd/4.0/).

https://doi.org/10.1016/j.xjtc.2020.06.023
}

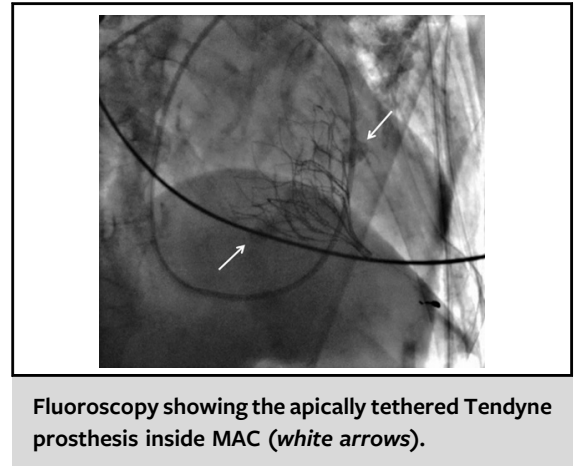

\begin{abstract}
CENTRAL MESSAGE
Enhanced intraoperative imaging could be useful in the performance of transapical transcatheter mitral valve implantation in the case of complex anatomies, such as mitral annular calcifications.
\end{abstract}

See Commentaries on pages 150 and 152.
Conventional surgery in the context of severe mitral annular calcification (MAC) has been associated with the risk of annular disruption and perivalvular leakage. Transcatheter mitral valve implantation (TMVI) using a transapically delivered, apically tethered, and self-expandable prosthesis (Tendyne device; Tendyne Holdings Inc, A Subsidiary of Abbott Laboratories, Chicago, Ill). This strategy has shown a 96\% technical success rate and a significant reduction in heart failure-related symptoms in patients with native mitral regurgitation. ${ }^{1}$ The presence of MAC was an exclusion criterion for the Tendyne Global Feasibility Study. Nonetheless, MAC represents an attractive field of application for this technology. TMVI in MAC using a balloon-expandable prosthesis has demonstrated inadequate immediate and delayed outcomes. ${ }^{2}$ In an initial experience, the Tendyne valve system showed encouraging results in mitral regurgitation associated with MAC. ${ }^{3}$

\section{CASE REPORT}

A 77-year-old woman had presented with advanced New York Heart Association class III dyspnea. Her history included left mastectomy and chest radiation therapy for a breast tumor. Transthoracic and transesophageal echocardiography revealed severe mitral regurgitation (Carpentier class IIIa, vena contracta width, $0.8 \mathrm{~cm}$; proximal isovelocity surface area effective regurgitant orifice area, $0.39 \mathrm{~cm}^{2}$ ). Her left ventricular ejection fraction and enddiastolic dimension were $60 \%$ and $49 \mathrm{~mm}$, respectively. The average transmitral gradient was $7 \mathrm{~mm} \mathrm{Hg}$. A mobile linear mass $(7 \times 3 \mathrm{~cm})$ was seen at the atrial side of the posterior annulus (Video 1). Active endocarditis was ruled out. The findings from a computed tomography (CT) scan confirmed significant MAC extending throughout the posterior annulus between the 2 trigones (Figure 1, $A$ ), with a uniform, smooth appearance and without irregular protrusion into the valve orifice. The patient was deemed to have excessive surgical risk; transcatheter edge-to-edge was not an option because the posterior leaflet was insufficient in length for grasping and because of the rigidity of the annulus due to MAC, predisposing to leaflet tear. TMVI using a Tendyne valve (size 27SP) was decided ("compassionate" indication), after sizing and calculation of a projected $317-\mathrm{mm}^{2}$ neo-left ventricular outflow tract in end-systole (Figure 1, B). We 


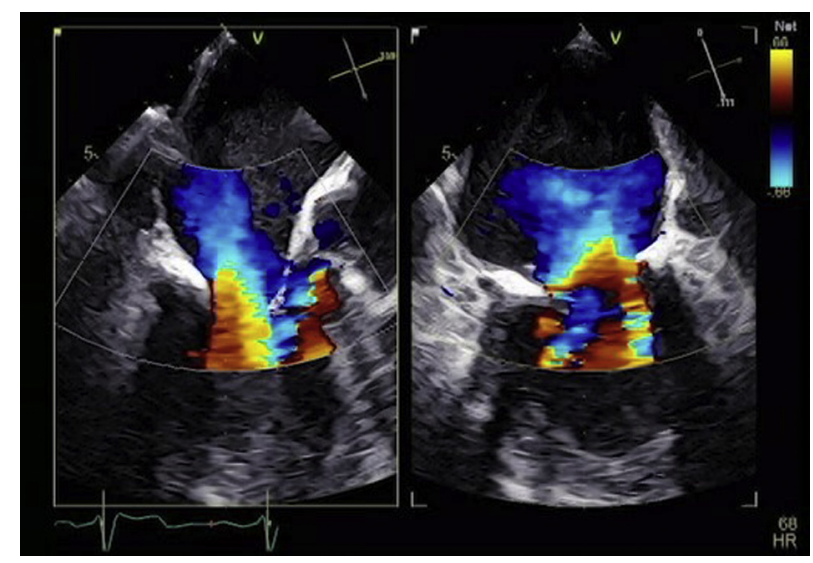

VIDEO 1. Preoperative transesophageal echocardiography imaging showing severe mitral regurgitation (Carpentier class IIIa) and a mobile linear mass at the level of the posterior mitral annulus. Video available at: https://www.jtcvs.org/article/S2666-2507(20)30301-1/fulltext.

expected that the posterior cuff of the valve would reliably cover the mobile mass and prevent embolism. The lesion was implanted $9 \mathrm{~mm}$ beyond the edge of MAC, constituting the posterior sealing zone (the posterior cuff of the device extends by $15 \mathrm{~mm}$ beyond).

The procedure was performed in a hybrid operative theater. The cardiac apex was approached through a left anterolateral minithoracotomy, and the $34 \mathrm{~F}$ delivery system was introduced transapically after catheterization of the left atrium. The valve was progressively deployed inside the native mitral annulus. In addition to echocardiography and fluoroscopic guidance, fusion imaging was used to overlay the echo representation of the mitral annulus/ MAC and baseline CT scan-derived images, on which the valve sizing and simulations had been performed (Figure 2 and Video 2). After valve deployment, its 3-dimensional echo representation was overlaid onto the CT scans. This provided (1) additional intraoperative guidance for valve deployment; and (2) confirmation of valve implantation in the intended position with respect to the annulus and MAC. Final postdeployment echocardiography showed absent mitral regurgitation, no perivalvular leakage, a 2-mm $\mathrm{Hg}$ average transvalvular gradient and actual coverage of the mobile mass. The postoperative course was uneventful, with no embolic episode reported. The patient was in New York Heart Association class II at 6 months after treatment. The present patient provided written informed consent for the management of their personal data for research purposes.

\section{DISCUSSION}

The present case illustrates the versatility of transapical TMVI for challenging anatomic conditions, including MAC and an increased gradient across the

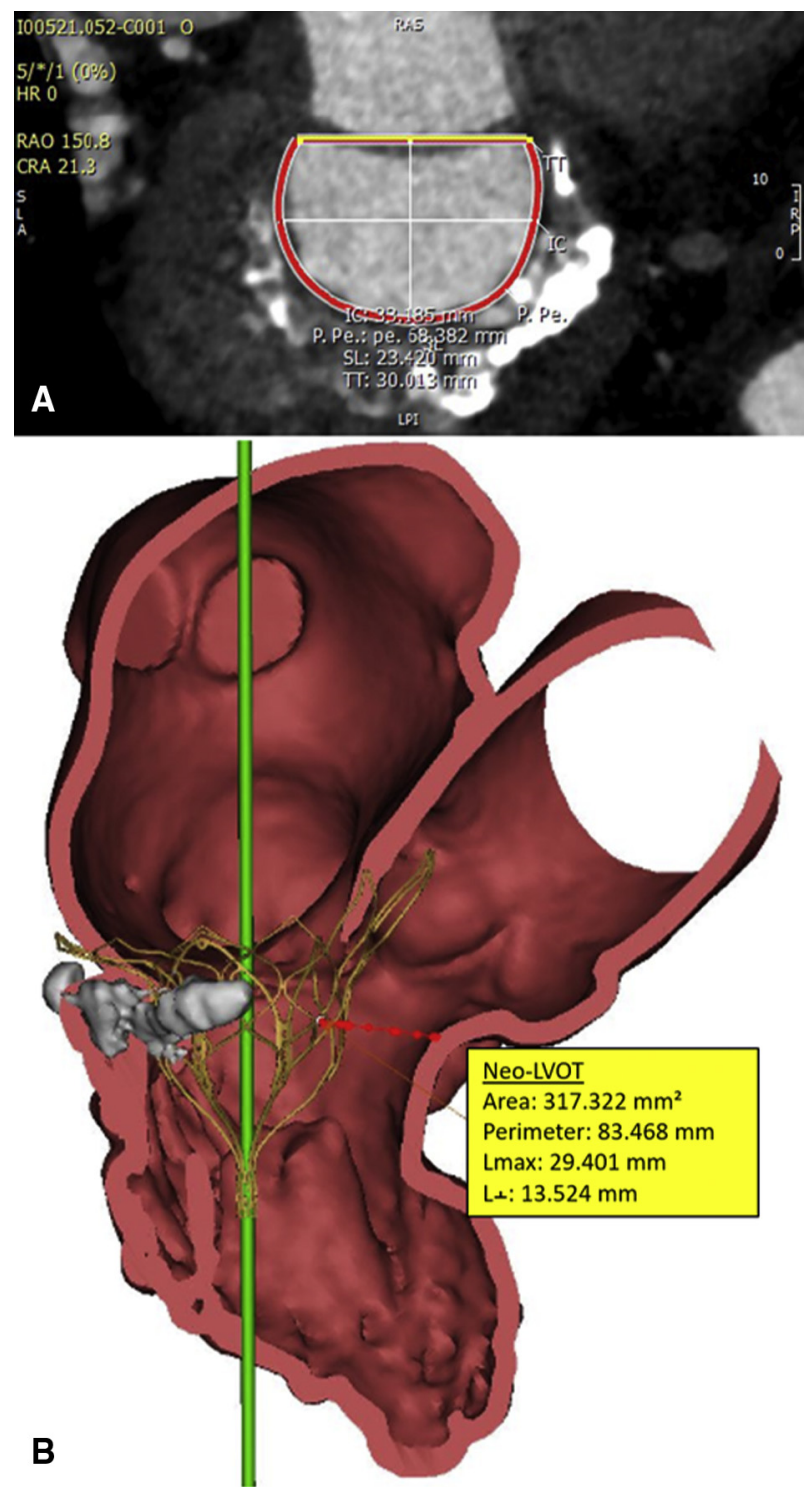

FIGURE 1. Sizing for Tendyne transcatheter mitral valve implantation in mitral annular calcification. A, Mitral annular sizing (perimeter, anteroposterior, and intercommissural distance) from contrast-enhanced computed tomography scan. B, Three-dimensional reconstructions from contrastenhanced computed tomography scan in end-systole. The frame of the selected valve prosthesis was virtually positioned to measure the expected left ventricular outflow tract (LVOT) clearance. Yellow insert shows measures of the projected neo-LVOT (surface, perimeter, maximum dimension, and orthogonal dimension). Lmax, Maximum length.

native mitral valve. Severe MAC results in an increased risk of death, complications, and dysfunction of mitral prosthesis after conventional surgery despite the standardization of strategies. ${ }^{4}$ Transcatheter transseptal TMVI-in-MAC using a balloon-expandable prosthesis (conceived for the treatment of aortic stenosis) has provided unsatisfactory results (rates of significant 


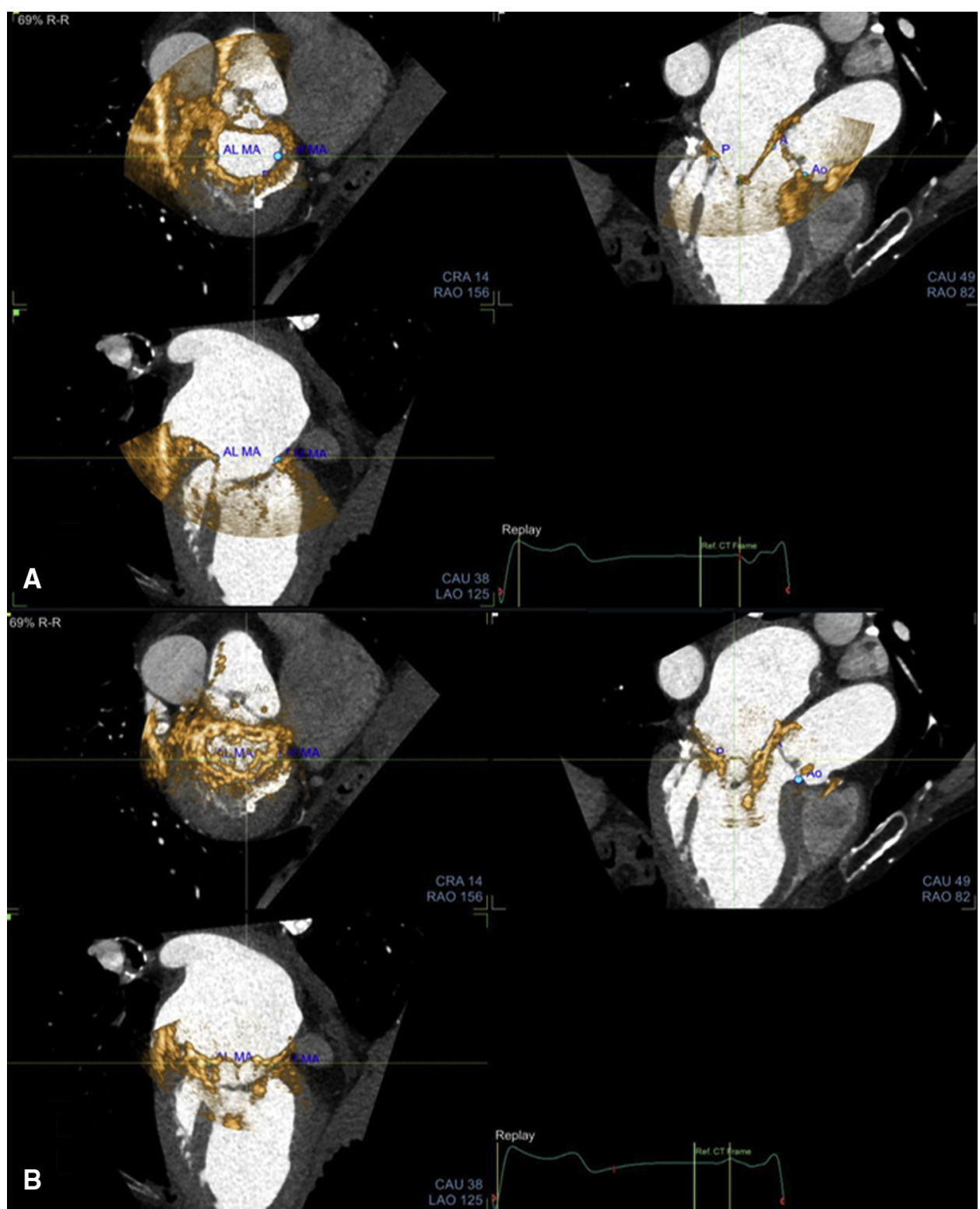

FIGURE 2. Fusion imaging (echocardiography and computed tomography) before (A) and after (B) transcatheter mitral valve implantation. An echo representation of the mitral annulus and mitral annular calcifications (A) or of the deployed Tendyne valve inside mitral annular calcifications (B) was overlain live during the procedure on computed tomography-derived images.

perivalvular leakage and migration). ${ }^{2}$ Additionally, left atrial thrombus or other potentially embolic lesions have been traditionally considered as contraindications to mitral interventions.

The Tendyne valve was originally developed as a TMVI device, with sealing obtained at the annular level (atrial cuff) and stability achieved through an apically secured tether (Figure 3). Although this procedure has proved reproducible, limited information is available about its performance in patients presenting with MAC. These constituted an exclusion criterion for the Tendyne Global Feasibility Study. Patients with MAC also represent a quite distinct subgroup, in whom valve sealing can be challenging and a risk of annular disruption exists. In the present patient, preoperative planning required additional simulation of the final valve position to evaluate the possibility of covering a lesion with the prosthesis cuff, potentially leading to embolism. Such a condition has been regarded as a contraindication to transcatheter mitral therapy. Fusion imaging provided additional intraoperative guidance (a representation of the MAC and native mitral annulus and, later, of the deployed valve was overlain onto the echocardiography and CT images). This facilitated verification of valve stability and positioning as 


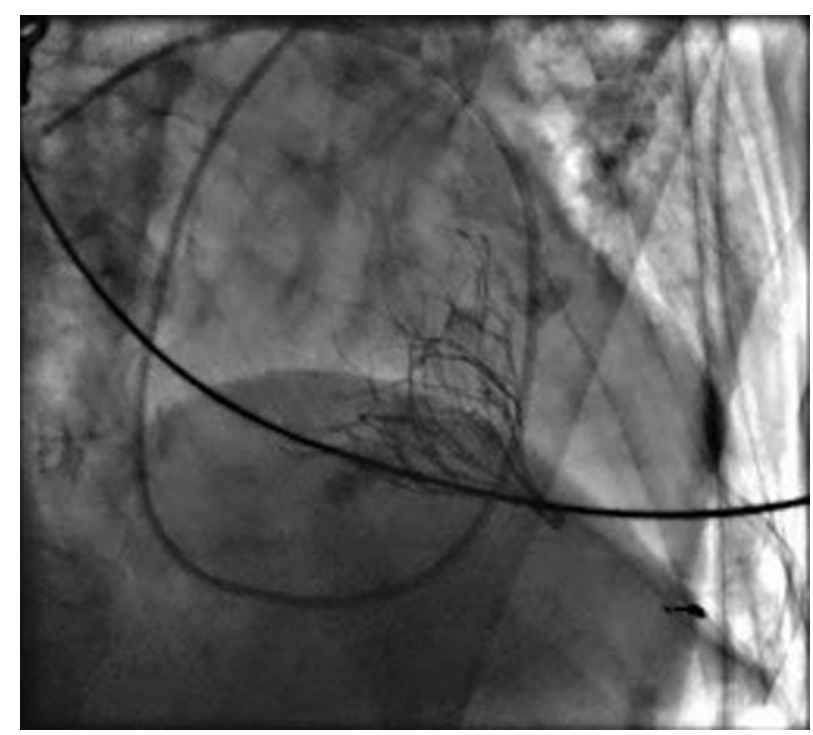

VIDEO 2. Intraoperative imaging (valve deployment, fusion imaging before and after deployment, final result on echocardiography). Video available at: https://www.jtcvs.org/article/S2666-2507(20)30301-1/fulltext.

planned compared with the preoperative CT scan, and of coverage of the mobile mass.

\section{CONCLUSIONS}

We suggest the versatility of TMVI with an apically tethered device in the context of MAC after careful preoperative planning. The design of this device, including a large intra-atrial cuff, can be exploited, not only to obtain sealing inside MAC, but also to manage lesions that could potentially lead to embolism located on the atrial side of the mitral valve.

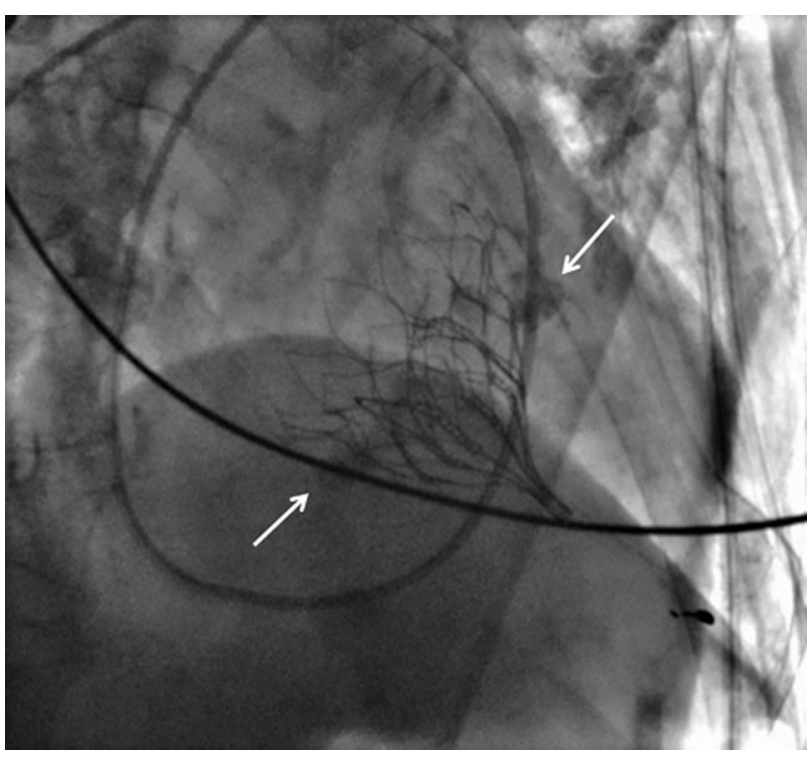

FIGURE 3. Fluoroscopy showing the apically tethered Tendyne prosthesis inside MAC (white arrows).

\section{References}

1. Sorajja P, Moat N, Badhwar V, Walters D, Paone G, Bethea B, et al. Initial feasibility study of a new transcatheter mitral prosthesis: the first 100 patients. $J \mathrm{Am}$ Coll Cardiol. 2019;73:1250-60.

2. Yoon SH, Whisenant BK, Bleiziffer S, Delgado V, Dhoble A, Schofer N, et al. Outcomes of transcatheter mitral valve replacement for degenerated bioprostheses, failed annuloplasty rings, and mitral annular calcification. Eur Heart J. 2019; 40:441-51.

3. Sorajja P, Gössl M, Babaliaros V, Rizik D, Conradi L, Bae R, et al. Novel transcatheter mitral valve prosthesis for patients with severe mitral annular calcification. J Am Coll Cardiol. 2019;74:1431-40.

4. Bedeir K, Kaneko T, Aranki S. Current and evolving strategies in the managemen of severe mitral annular calcification. J Thorac Cardiovasc Surg. 2019;157: 555-66. 